\title{
Is islet autoimmunity related to insulin sensitivity or body weight in children of parents with type 1 diabetes?
}

\author{
C. Winkler • S. Marienfeld $\cdot$ M. Zwilling $\cdot$ E. Bonifacio • \\ A.-G. Ziegler
}

Received: 7 January 2009 / Accepted: 12 June 2009 /Published online: 30 July 2009

(C) Springer-Verlag 2009

\begin{abstract}
Aims/hypothesis It has been suggested that metabolic demand and insulin resistance play a role in the development of type 1 diabetes, including the onset of autoimmunity. The aim of the present study was to determine whether insulin demand is increased in children with islet autoantibodies.

Methods BMI standard deviation score (BMI-SDS) was measured from 2 years of age in 1,650 prospectively followed children of mothers or fathers with type 1 diabetes, including 135 who developed persistent islet autoantibodies. HOMA of insulin resistance (HOMA-IR) was determined using fasting samples from 777 of the children starting from age 5 years.

Results An increased HOMA-IR was associated with female sex $(p=0.0004)$, older age $(p<0.0001)$ and increased BMI-SDS $(p<0.0001)$. Children with islet autoantibodies did not have an increased HOMA-IR compared with agematched islet autoantibody-negative children (age 8 years:
\end{abstract}

C. Winkler $\cdot$ S. Marienfeld $\cdot$ M. Zwilling $\cdot$ A.-G. Ziegler

Institut für Diabetesforschung der Forschergruppe Diabetes e.V.,

Helmholtz Center Munich,

Neuherberg, Germany

E. Bonifacio

Center for Regenerative Therapies-Dresden University of

Technology, Biotechnology Center Tatzberg,

Dresden, Germany

A.-G. Ziegler $(\bowtie)$

Forschergruppe Diabetes der Technische Universität München,

Koelner Platz 1,

80804 Munich, Germany

e-mail: anziegler@1rz.uni-muenchen.de

A.-G. Ziegler

Klinik für Endokrinologie, Diabetologie und Suchtmedizin,

Klinikum Schwabing StKM,

Munich, Germany mean 0.61 vs mean 0.72 , respectively, $p=0.21$; age 11 years: mean 0.96 vs mean 1.21, respectively, $p=0.07$ ). Furthermore, after correction for age and sex, autoantibody positivity was associated with decreased HOMA-IR values $(p=0.01)$. BMI-SDS was similar between islet autoantibodypositive and -negative children at age 2 (mean 0.07 vs mean 0.16 , respectively), 5 (mean 0.06 vs 0.08 , respectively), 8 (mean -0.09 vs mean 0.02 , respectively), and 11 years (mean 0.22 vs mean 0.16 , respectively) and similar to that of national reference values.

Conclusions/interpretation Islet autoantibody-positive children in the BABYDIAB cohort are not insulin resistant and do not have an increased BMI around and early after islet autoantibody seroconversion. These findings are inconsistent with the notion that insulin resistance is a risk factor for islet autoimmunity.

Keywords Body weight - Insulin resistance . Islet autoimmunity

$\begin{array}{ll}\text { Abbreviations } \\ \text { islet ab } & \text { Islet autoantibody } \\ \text { BMI-SDS } & \text { BMI standard deviation score } \\ \text { GADA } & \text { GAD autoantibodies } \\ \text { HOMA-IR } & \text { HOMA of insulin resistance } \\ \text { IAA } & \text { Insulin autoantibodies } \\ \text { IA2A } & \text { Tyrosine phosphatase autoantibodies }\end{array}$

Introduction

Type 1 diabetes is a chronic autoimmune disease caused by the selective destruction of pancreatic islets, which leads to insulin deficiency, but the aetiology of type 1 diabetes is 
still unknown [1-3]. Roles for impaired insulin action and increased insulin demand in the pathogenesis of autoimmune type 1 diabetes have been proposed $[4,5]$. There are consistent reports indicating that decreased insulin sensitivity leads to the earlier development of diabetes in islet autoantibody-positive relatives, particularly when they show a concomitant decrease in beta cell function [6-8]. Moreover, a causal aetiological role for insulin sensitivity in driving beta cell destruction, or indeed, initiating islet autoimmunity, has been suggested, but no direct evidence has been reported. Indirect evidence for this comes from reports showing that HLA genotypes associated with a high risk for type 1 diabetes are linked with birthweight, and from epidemiological studies indicating that the incidences of obesity and type 1 diabetes are increasing in parallel in childhood [9-13]. Stronger evidence would be provided if insulin demand was found to be increased in the prediabetic period, but thus far no direct comparison of insulin sensitivity between autoantibody-positive and -negative individuals has been reported. To address this, we examined BMI and HOMA of insulin resistance (HOMA-IR, a marker of insulin sensitivity) in autoantibody-positive and -negative children participating in the BABYDIAB study. Specifically, the aims of this study were to determine whether children with islet autoantibodies have a lower insulin sensitivity and/or are more obese than children who remain autoantibody negative.

\section{Methods}

Participants The BABYDIAB study examines the natural history of autoimmunity to islet antigens from birth in the children of parents with type 1 diabetes [14]. Families were eligible to participate if one or both parents had type 1 diabetes. Recruitment into the study began in 1989 and ended in 2000. Venous blood samples from the child during follow-up were requested at scheduled study visits at age 9 months and 2, 5, 8, 11, 14 and 17 years. For most visits, samples were obtained within the requested time window for each study visit (6-12 months, 1.5-2.5 years, 4.55.5 years, $7.5-8.5$ years, $10.5-11.5$ years, $13.5-14.5$ years, 16.5-17.5 years). Some children attended study visits outside these time windows. Islet autoantibodies were measured in samples taken at all scheduled visits. If children had a positive autoantibody finding, they were asked to provide a sample for confirmation of autoantibody status within 6 months. Questionnaires were completed by the parents at the birth of each child and at each paediatric visit, at which time the child also underwent a physical examination performed by the paediatrician. The study was coordinated by the Diabetes Research Institute in Munich (Institut für Diabetesforschung der Forschergruppe Diabetes
e.V. at the Helmholtz Center Munich) through direct contact with the families and the family paediatrician. Offspring were considered as participants of the BABYDIAB study if they had been recruited at birth and had participated in at least the 9 month follow-up visit. A total of 1,650 offspring met these criteria. The median follow-up time from birth to last sample was 8.29 years (range $0.75-18.5$ years). The cumulative dropout rate was $16 \%$ by age 5 years and $20.9 \%$ by age 8 years. All families gave written informed consent to participate in the BABYDIAB study. The study was approved by the ethical committee of Bavaria, Germany (Bayerische Landesärztekammer number 95357).

Outcome definitions Islet autoimmunity was defined by the appearance of islet autoantibodies. Offspring were considered islet autoantibody-positive if at least two consecutive samples collected after birth were found to be positive for one or more islet autoantibodies (to insulin [IAA], GAD (GADA) or tyrosine phosphatase-like protein [IA2A]). Positivity for more than one islet autoantibody (multiple islet autoantibody-positive) was used as a secondary outcome. A total of 135 children had at least one persistent islet autoantibody, and 61 of these children had multiple islet autoantibodies. Five children who were islet autoantibody-positive on one occasion and who did not provide a follow-up sample for confirmation of autoantibody status were not included in the analyses.

$B M I$ Weight and height were collected from paediatric records at birth and at ages 9 months and 2, 5, 8, 11, 14 and 17 years. Paediatric records were completed by trained staff at delivery and by paediatricians at visits to the clinic after birth. Data on height, weight and BMI at follow-up were adjusted for sex and exact age at examination; BMI was expressed as the BMI standard deviation score (BMI-SDS) [15], with reference to the national data provided by the study group Adipositas im Kinders- und Jugendalter [16]. BMI data were available for 1,414 children at age 2 years, 1,294 children at 5 years, 791 children at 8 years, 434 children at 11 years, 120 children at age 14 years and ten children at 17 years (Table 1).

HOMA-IR Blood samples for the determination of HOMA-IR had been collected since 2003 from children aged 5 years and above. Children were asked to fast for at least $8 \mathrm{~h}$ before blood collection. Fasting blood glucose was determined locally by the pathology laboratory at the paediatric outpatient department, and the blood glucose value was sent to the Diabetes Research Institute. Serum samples for the measurement of the fasting insulin were sent by express courier from the paediatrician to the Diabetes Research Institute. For this analysis, data were available for 25 children at age 5 years, 482 children at 8 years, 344 children at 11 years, 85 children at 
Table 1 BMI-SDS and HOMAIR at each study visit age
${ }^{\text {a }}$ Numbers refer to children who have reached the follow-up visit age and are still in the study at the time of analysis (June 2008)

\begin{tabular}{llll}
\hline Age & Islet autoantibody status & Cases with BMI-SDS $(n)$ & Cases with HOMA-IR $(n)$ \\
\hline 2 years $(n=1,511)^{\mathrm{a}}$ & ${\text { islet } \mathrm{ab}^{+} \text {at visit }}^{2}$ & 50 & 0 \\
& islet $\mathrm{ab}^{+}$subsequently & 69 & 0 \\
& islet $\mathrm{ab}^{-}$ & 1,295 & 0 \\
5 years $(n=1,372)^{\mathrm{a}}$ & islet $\mathrm{ab}^{+}$at visit & 76 & 23 \\
& islet $\mathrm{ab}^{+}$subsequently & 35 & 0 \\
& islet $\mathrm{ab}^{-}$ & 1,183 & 2 \\
8 years $(n=1,116)^{\mathrm{a}}$ & islet $\mathrm{ab}^{+}$at visit & 63 & 44 \\
& islet $\mathrm{ab}^{+}$subsequently & 9 & 0 \\
& islet $\mathrm{ab}^{-}$ & 719 & 438 \\
11 years $(n=522)^{\mathrm{a}}$ & islet $\mathrm{ab}^{+}$at visit & 38 & 36 \\
& islet $\mathrm{ab}^{+}$subsequently & 3 & 1 \\
& islet $\mathrm{ab}^{-}$ & 393 & 307 \\
14 years $(n=132)^{\mathrm{a}}$ & islet $\mathrm{ab}^{+}$at visit & 33 & 17 \\
& islet $\mathrm{ab}^{+}$subsequently & 0 & 0 \\
& islet $\mathrm{ab}^{-}$ & 87 & 68 \\
17 years $(n=10)^{\mathrm{a}}$ & islet $\mathrm{ab}^{+}$subsequently & 3 & 1 \\
& islet $\mathrm{ab}^{+}$at follow-up & 0 & 0 \\
& islet $\mathrm{ab}^{-}$ & 7 & 5 \\
\hline
\end{tabular}

14 years and six children at 17 years; 165 children had more than one measurement available for analysis (Table 1). Fasting insulin was determined using the Mercodia Ultrasensitive Insulin ELISA (Uppsala, Sweden), a solid-phase two-site enzyme immunosorbent assay, as described in the manufacturer's instructions. Insulin resistance was estimated by the HOMA of insulin resistance (HOMA-IR) [17].

Islet autoantibodies IAA, GADA and IA-2 (IA2A) were determined as previously described [14]. The upper limits of normal corresponded to the 99th percentile of the control children, and were 8.5 local units $/ \mathrm{ml}$ or $25 \mathrm{WHO}$ units $/ \mathrm{ml}$ for GADA, 2.5 local units $/ \mathrm{ml}$ or $4 \mathrm{WHO}$ units $/ \mathrm{ml}$ for IA2A, and 1.5 local units/ml for IAA.

Statistical analysis Relationships between HOMA-IR and age, sex and BMI-SDS were analysed by linear regression using the first available HOMA-IR measurement for each child. For specific comparisons of HOMA-IR levels at age 8 years and at age 11 years between islet autoantibodypositive and -negative children, analysis of variance methods were used; for this analysis, visits that occurred within the requested time window for 8 and 11 years were used. For all analyses, logarithmically transformed $\left(\log _{\mathrm{e}}\right)$ values of HOMA-IR were used so that assumptions of the general linear model and parametric analyses were met. Accordingly, means and 95\% confidence limits reported for HOMA-IR are based on log-transformed data. At each visit age, BMI-SDS values were compared between children with and without islet autoimmunity (and with and without multiple autoantibodies) by analysis of variance. For all analyses, a two-tailed $p$ value of 0.05 was considered significant. All statistical analyses were performed using the Statistical Package for Social Science (SPSS 16.0; SPSS, Chicago, IL, USA).

\section{Results}

HOMA-IR and age, sex, BMI-SDS HOMA-IR was significantly higher in girls than boys (mean 0.96 vs mean 0.86 , $p=0.0004$; Fig 1a). An increased HOMA-IR was associated with an increased BMI-SDS $(r=0.24, p<0.0001)$ and there was a significant relationship between HOMA-IR and age $(r=0.32, p<0.0001$; Fig. $1 \mathrm{~b}$ and $\mathrm{c})$.

HOMA-IR and islet autoimmunity Of 777 children with HOMA-IR measurements, 84 had developed confirmed islet autoantibodies during the study. Analysis of HOMAIR by age showed no significant difference in HOMA-IR between islet autoantibody-positive and islet autoantibodynegative children, with a tendency towards a lower HOMAIR in the antibody-positive children compared with the antibody-negative children (age 8 years: mean 0.61 , [95\% CI 0.49-0.77] vs mean 0.72 [95\% CI 0.67-0.79], $p=0.21$; age 11 years: mean 0.96 [95\% CI $0.70-1.31$ ] vs mean 1.21 [95\% CI 1.20-1.30], $p=0.07$; Fig. 2a,b). Moreover, when all children with HOMA measurements were considered collectively using their first available HOMA-IR measurement as the dependent variable, islet autoantibody positivity was associated with significantly lower HOMA-IR 


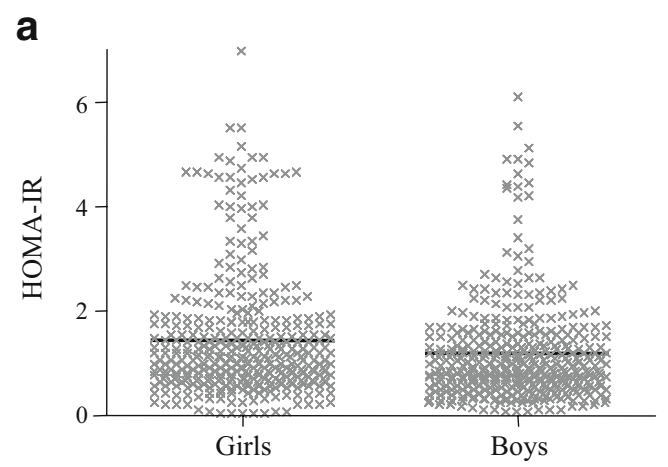

b

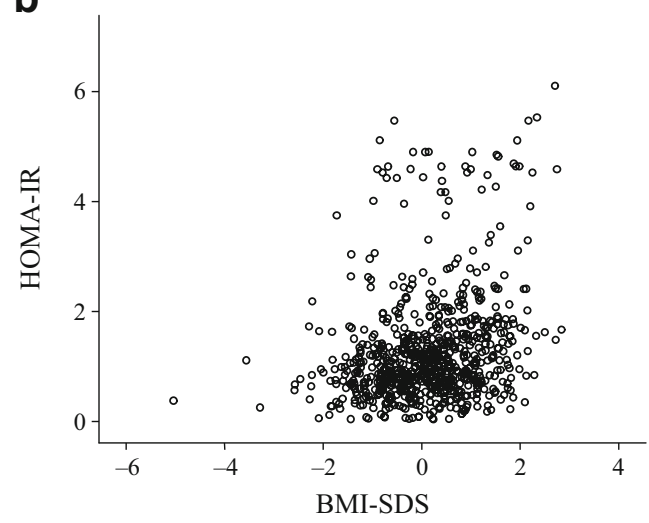

C

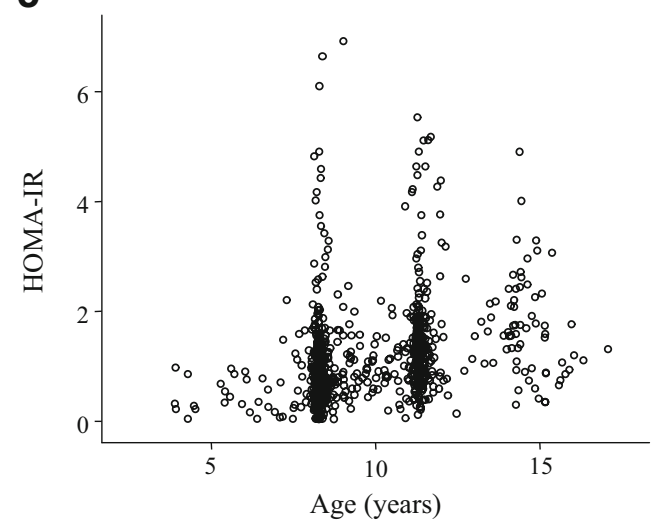

Fig. 1 a Comparison of HOMA-IR between girls and boys ( $p=$ $0.0004)$. The horizontal lines indicate the mean. b Correlation between HOMA-IR and BMI-SDS $(p<0.0001)$. $\mathbf{c}$ Correlation between HOMAIR and age $(p<0.0001)$

$(p=0.01)$ after correction for age at time of measurement and sex.

BMI and islet autoimmunity BMI was measured at least once from age 2 years in 1,650 children, including 135 who had developed islet autoantibodies by the time of analysis. Islet autoantibody-positive children did not have an increased BMI-SDS as compared with islet autoantibodynegative children at age 2, 5, 8 or 11 years (Fig. 3). Among the antibody-positive children, BMI-SDS was similar in children who were already islet autoantibody-positive at
BMI-SDS measurement and those who subsequently developed autoantibodies. Furthermore, no differences were observed when the analysis was restricted to children with multiple islet autoantibodies (data not shown).

\section{Discussion}

Increased insulin demand has been suggested to have an important role in the pathogenesis of autoimmunity in type 1 diabetes [4-8]. In the present study we aimed to obtain evidence supporting this hypothesis by analysing two known causes of insulin demand, BMI and HOMA-IR (as a marker of insulin sensitivity), in islet autoantibodypositive and -negative children who were prospectively followed from birth. Neither BMI nor HOMA-IR was increased in the islet autoantibody-positive children at any age. Thus, we were unable to find evidence to support an increased insulin demand in children who develop islet autoantibodies.

This is a prospective study in which measurements of islet autoantibodies, BMI and HOMA-IR are performed in real time. BMI was analysed from the age of 2 years in these children, and HOMA-IR was determined from as early as 5 years of age. HOMA-IR measurements were available for over 700 children who provided fasting blood samples during the course of the study. All insulin measurements were performed centrally, whereas blood glucose measurements were performed locally by a pathology laboratory. The measurements of HOMA-IR were consistent with previous reports showing a correlation between HOMAIR and BMI [18-21]. We found that girls had an increased HOMA-IR compared with boys. This difference between the sexes with respect to insulin resistance has also been a

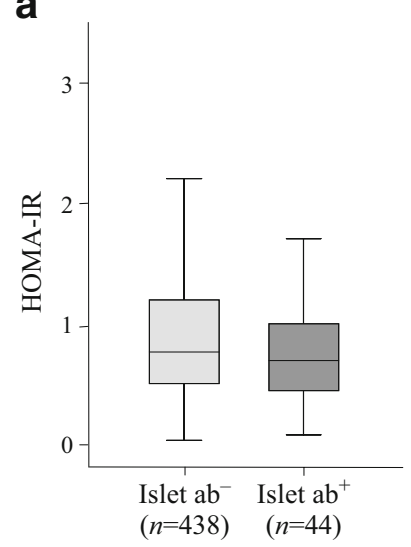

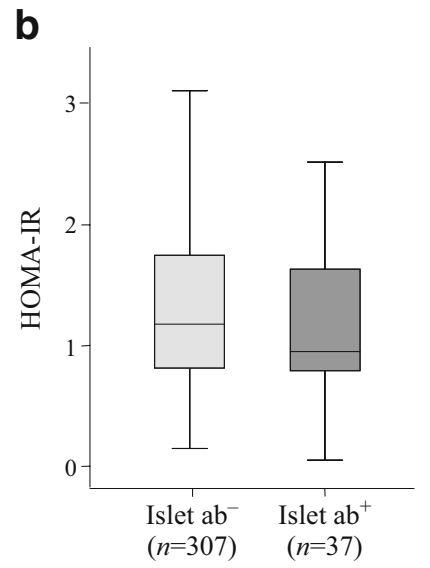

b
Fig. 2 Comparison of HOMA-IR between islet autoantibody-positive and -negative children at age 8 years $(p=0.21)$ (a) and 11 years $(p=$ 0.07) (b). The box and whisker plots show the median, interquartile range and 1.5 interquartile range 
a

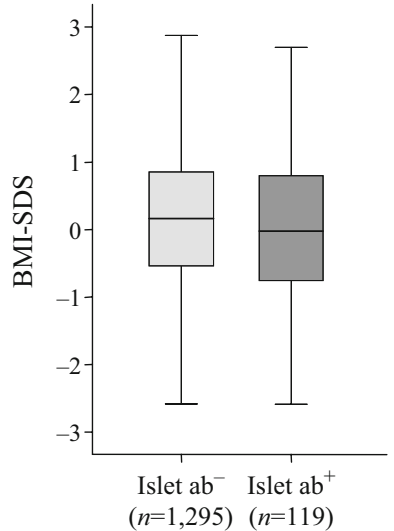

C

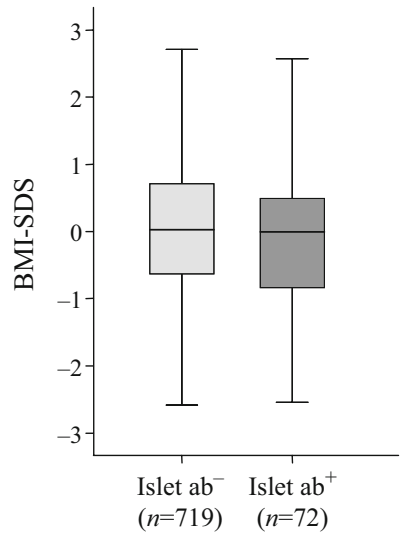

b

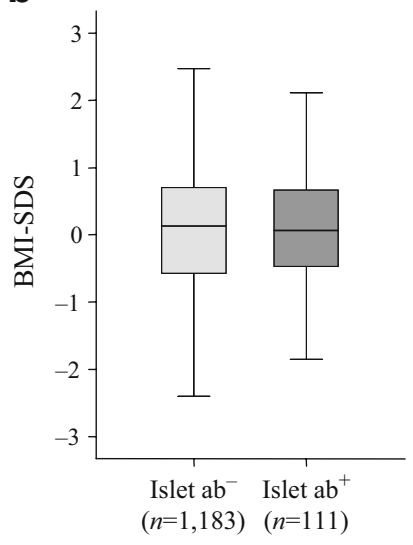

d

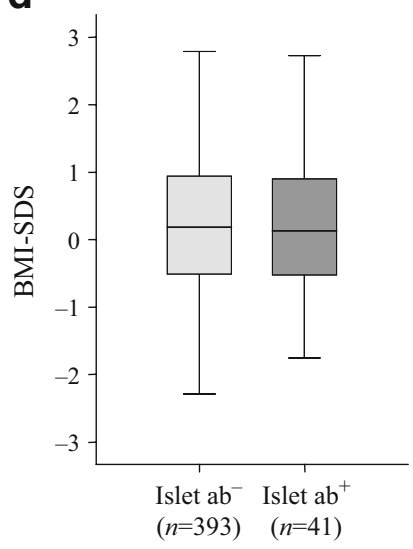

Fig. 3 Comparison of BMI-SDS between islet autoantibody-positive and -negative children at age 2 years $(p=0.22)(\mathbf{a}), 5$ years $(p=0.69)$ (b), 8 years $(p=0.55)(\mathbf{c})$ and age 11 years $(p=0.78)(\mathbf{d})$. The box and whisker plots show the median, interquartile range and 1.5 interquartile range

documented in other studies and is likely to be due to differences in fat deposition and distribution and pubertal age between boys and girls [20, 21]. Finally, an increased HOMA-IR was associated with older age in our study cohort of children between 5 and 17 years of age. Previous studies have also reported an association between insulin resistance and age, especially with the onset of puberty. It is postulated that growth hormones and an increased body mass contribute to this relationship [20, 21]. Unfortunately, Tanner stages were only available for a few of the antibody-positive children who participated in our study.

Together with the many merits of the measurements performed in the study, some limitations need to be considered when interpreting the data. First, HOMA-IR is only a surrogate measure of insulin sensitivity, and the relationship between HOMA-IR and insulin sensitivity measured by sophisticated clamp measurements is not perfect [22]. Thus, the relationships between insulin sensitivity and autoantibody status might differ if measurements were performed using more accurate measurements

of insulin sensitivity. A second limitation is that, for most of the autoantibody-positive children, the first HOMA-IR measurements were obtained when the child had developed autoantibodies. Thus, although HOMA-IR was determined early in the history of islet autoimmunity, and in a number of cases, prior to the development of islet autoantibodies, we cannot exclude the possibility that HOMA-IR may have been transiently increased shortly prior to the development of islet autoantibodies. Similarly, 30 children developed diabetes prior to of the commencement of HOMA-IR determination, and we cannot exclude the possibility that these children were more likely to be insulin resistant than children who developed diabetes later. A potential limitation is that some of the islet autoantibody-negative children are likely to develop autoantibodies at a subsequent time point. The number is expected to be very small, and this is unlikely to greatly influence the results. The missing data for children who either dropped out of the study or did not provide samples for HOMA-IR measurement may also have biased the findings. We were unable to determine the time course of HOMA-IR, since multiple HOMA-IR measurements were obtained in less than half of the children analysed. Finally, since the data are from children with a family history of type 1 diabetes, the HOMA-IR may not reflect those seen in children from unaffected families. Note, however, that the BMI for the children in this study was not different from that observed in a reference German population [15].

A link between insulin demand and autoimmunity would support converging pathogenetic mechanisms in type 1 and type 2 diabetes [4], but current evidence for this link is largely circumstantial. While there are some data suggesting that birthweight differs according to HLA genotype, including increased birthweight in children with certain type 1 diabetes-susceptibility HLA alleles [9-11], there is no clear relationship between birthweight and risk of type 1 diabetes $[12,13]$. Moreover, in our BABYDIAB study, the highest risks for autoimmunity appeared to be associated with normal birthweight [23]. There have also been reports of associations between BMI and autoantibody concentration, but these studies had very few autoantibody-positive cases, and the correlations were made between BMI and antibody concentrations that were largely at the upper end of the normal range, values that are extremely difficult to reproduce in multiple laboratories, i.e. potentially noise in the autoantibody assays [24].

Our current study is perhaps the most informative with respect to testing the accelerator hypotheses, and shows no association between either increased HOMA-IR or BMI and autoantibody positivity. Indeed, the autoantibodypositive children had a slight decrease in HOMA-IR as compared with the islet autoantibody-negative children. The study also shows that, at least in Germany, 
autoantibody-positive children are neither excessively overweight nor insulin resistant. Thus, a substantial contribution of insulin demand on progression to type 1 diabetes is unlikely to be true for the majority of autoantibody-positive children over the last decade. This may change in the future if obesity continues to increase. We believe it is likely that (1) islet autoimmunity develops on a background of genetic susceptibility regardless of insulin demand; (2) the eventual development or not of type 1 diabetes is unaffected by insulin demand; but (3) insulin demand will affect the timing of clinical presentation of diabetes if beta cell mass or function is reduced. This last point is supported by data from other studies [6-8] that show a clear relationship between insulin resistance measured during IVGTT tests and the development of clinical diabetes if beta cell function is considered in the statistical model. The notion that obesity and insulin demand do not affect the presentation of autoimmunity is also supported by animal models in which severely obese mice that were genetically susceptible to type 1 diabetes rarely developed the disease $[25,26]$. Indeed, it appears that the obesity protected from the development of autoimmunity in these mice, although it is possible that the leptin receptor mutation introduced into the mice may have conferred protection independently from the obesity.

In conclusion, while prevention or delay of type 1 diabetes may be achieved by including therapeutic agents that increase insulin sensitivity or beta cell function, it is unlikely that such therapies will be effective in preventing the initiation of autoimmunity or progression of autoimmunity early in the pathogenesis of the disease.

Acknowledgements The study was supported by the Juvenile Diabetes Research Foundation (JDRF no. 1-2006-665) and the Kompetenznetz Diabetes mellitus (Competence Network for Diabetes mellitus) funded by the Federal Ministry of Education and Research (FKZ 01GI0805-07). We thank A. Knopff, A. Jäger, U. Mollenhauer and M. Walter (at the Institut für Diabetesforschung der Forschergruppe Diabetes e.V. at the Helmholtz Center Munich) for expert technical assistance. We also thank all paediatricians and family doctors in Germany for participation in the BABYDIAB study.

Duality of interest The authors declare that there is no duality of interest associated with this manuscript.

\section{References}

1. Achenbach P, Bonifacio E, Koczwara K, Ziegler AG (2005) Natural history of type 1 diabetes. Diabetes 54(Suppl 2):S25-31

2. Atkinson MA, Eisenbarth GS (2001) Type 1 diabetes: new perspectives on disease pathogenesis and treatment. Lancet 358:221-229

3. Gianani R, Eisenbarth GS (2005) The stage of type 1A diabetes: 2005. Immunol Rev 204:232-249

4. Wilkin TJ (2001) The accelerator hypothesis: weight gain as the missing link between type 1 and type 2 diabetes. Diabetologia 44:914-922
5. Dahlquist G (2006) Can we slow the rising incidence of childhood-onset autoimmune diabetes? The overload hypothesis. Diabetologia 49:20-24

6. Fourlanos S, Narendran P, Byrnes GB, Colman PG, Harrison LC (2004) Insulin resistance is a risk factor for progression to type 1 diabetes. Diabetologia 47:1661-1667

7. Bingley PJ, Mahon JL, Gale EAM, for the European Nicotinamide Diabetes Intervention Trial (ENDIT) Group (2008) Insulin resistance and progression to type 1 diabetes in the European Nicotinamide Diabetes Intervention Trial (ENDIT). Diabetes Care $31: 146-150$

8. Xu P, Cuthbertson D, Greenbaum C, Palmer JP, Krischer JP, for the Diabetes Prevention Trial-Type 1 Study Group (2007) Role of insulin resistance in predicting progression to type 1 diabetes. Diabetes Care 30:2314-2320

9. Larsson HE, Lynch K, Lernmark B et al (2005) Diabetes associated HLA genotypes affect birthweight in the general population. Diabetologia 48:1484-1491

10. Järvinen TM, Harjutsalo V, Kinnunen L, Miettinen ME, Tuomilehto-Wolf E, Tuomilehto J (2008) A population-specific diabetogenic haplotype HLA-A2, Cw1, B56, DR4, DQ8 is associated with high birthweight in Finnish diabetic families. Genes Immun 9:207-213

11. Hummel M, Marienfeld S, Huppmann M et al (2007) Fetal growth is increased by maternal type 1 diabetes and HLA DR4-related gene interactions. Diabetologia 50:850-858

12. Knip M, Reunanen A, Virtanen SM, Nuutinen M, Viikari J, Akerblom HK (2008) Does the secular increase in body mass in children contribute to the increasing incidence of type 1 diabetes? Pediat Diabetes 9:46-49

13. Hyppönen E, Virtanen SM, Kenward MG, Knip M, Akerblom HK, the Childhood Diabetes in Finland Study Group (2000) Obesity, increased linear growth, and risk of type 1 diabetes in children. Diabetes Care 23:1755-1760

14. Ziegler AG, Hummel M, Schenker M, Bonifacio E (1999) Autoantibody appearance and risk for development of childhood diabetes in offspring of parents with type 1 diabetes: the 2-year analysis of the German BABYDIAB Study. Diabetes 48:460-468

15. Kromeyer-Hauschild K, Wabitsch M et al (2001) Perzentile für den body-mass-index für das kinder- und jugendalter unter heranziehung verschiedener stichproben. Monatsschr Kinderheilkd 8:807-818; article in German

16. Arbeitsgemeinschaft adipositas im kindes- und jugendalter: bodymass-index für deutsche kinder und jugendliche. Available from www.mybmi.de, accessed 20 March 2008

17. Matthews DR, Hosker JP, Rudenski AS, Naylor BA, Treacher DF, Turner RC (1985) Homeostasis model assessment: insulin resistance and beta-cell function from fasting plasma glucose and insulin concentrations in man. Diabetologia 28:412-419

18. Steinberger J, Daniels SR (2003) Obesity, insulin resistance, diabetes, and cardiovascular risk in children: an American Heart Association scientific statement from the Atherosclerosis, Hypertension, and Obesity in the Young Committee (Council on Cardiovascular Disease in the Young) and the Diabetes Committee (Council on Nutrition, Physical Activity, and Metabolism). Circulation 107:1448-1453

19. Dietz WH, Robinson TN (1998) Use of the body mass index (BMI) as a measure of overweight in children and adolescents. J Pediatr 132:191-193

20. Szadkowska A, Pietrzak I, Mianowska B et al (2008) Insulin sensitivity in type 1 diabetic children and adolescents. Diabetic Medicine 25:282-288

21. Moran A, Jacobs DR, Steinberger J et al (1999) Insulin resistance during puberty. Diabetes 48:2039-2044

22. Brandou F, Brun JF, Mercier J (2005) Limited accuracy of surrogates of insulin resistance during puberty in obese and lean 
children at risk for altered glucoregulation. J Clin Endocrinol Metab 90:761-767

23. Bonifacio E, Pflüger M, Marienfeld S, Winkler C, Hummel M, Ziegler AG (2008) Maternal type 1 diabetes reduces the risk of islet autoantibodies: relationships with birthweight and maternal $\mathrm{HbA}_{1 \mathrm{c}}$. Diabetologia 51:1245-1252

24. Rolandsson O, Hägg E, Hampe C et al (1999) Glutamate decarboxylase (GAD65) and tyrosine phosphatase-like protein (IA-2) autoantibodies index in a regional population is related to glucose intolerance and body mass index. Diabetologia 42:555559

25. Lee CH, Reifsnyder PC, Naggert JK et al (2005) Novel leptin receptor mutation in NOD/LtJ mice suppresses type 1 diabetes progression: I. Pathophysiological analysis. Diabetes 54:25252532

26. Lee CH, Chen YG, Chen J et al (2006) Novel leptin receptor mutation in NOD/LtJ mice suppresses type 1 diabetes progression: II. Immunologic analysis. Diabetes 55:171-178 